\title{
SOLVABLE GROUPS AND QUADRATIC FORMS
}

\author{
BY \\ RICHARD TOLIMIERI
}

\begin{abstract}
A solvable Lie group and a unitary representation are constructed from a given binary quadratic form. The multiplicity of this representation is related to the arithmetic of the form.
\end{abstract}

1. Introduction. In this paper we relate various classical problems in the theory of quadratic forms and in the theory of unitary representation theory of solvable groups. Let us give some indication of the problems we have in mind. In the following section these problems will be made more precise.

Let $F\left(X_{1}, X_{2}\right)=\alpha X_{1}^{2}+\beta X_{1} X_{2}+\gamma X_{2}^{2}$ be an integral binary quadratic form. We are primarily concerned with the case when $F\left(X_{1}, X_{2}\right)$ is a nondegenerate form whose discriminant is not a perfect square. The reasons for this restriction will be made clear below. For an integer $v$ it is a classical problem to investigate the integral solutions $n=\left(n_{1}, n_{2}\right) \in Z^{2}$ of the equation $F\left(X_{1}, X_{2}\right)=v$. The number of 'nonassociated' integral solutions of this equation has been of central interest in the study of quadratic forms. To be concise we shall call this problem the multiplicity problem of the form $F\left(X_{1}, X_{2}\right)$. As is well known, this problem is closely related to the problem of computing the class number of a quadratic field.

Let $S$ be a solvable Lie group. We consider a unitary representation denoted by $R$ of $S$ on some Hilbert space $V$. In the situation we have in mind, $V$ decomposes into a countable direct sum $V=\Sigma_{n \in Z} \bigoplus V_{n}$ of R-invariant and irreducible subspaces $V_{n}$ of $V$. We make this assumption in all that follows. An arbitrary irreducible representation $S$ of $S$ is said to occur in $R$ whenever there is an $R$-invariant and irreducible subspace $W$ of $V$ such that $S$ is unitarily equivalent to $R \mid W$. By $R \mid W$ we mean the unitary representation of $S$ defined by restricting the operators $R_{S}$ of $V$ to the space $W$. In any direct sum decomposition of $V$ into $R$-invariant and irreducible subspaces, the number of times a given irreducible representation of $S$ occurs as a direct summand is independent of the direct sum decomposition. We call this number the multiplicity of the given irreducible representation in $R$. In

Received by the editors February 14, 1974.

AMS (MOS) subject classifications (1970). Primary 12A25, 12Axx, 22D10, 12Dxx.

Key words and phrases. Solvable groups, unitary representation, quadratic form, multiplicity. 
general, it may be infinite, but in our cases it will always be finite. The multiplicity problem for the unitary representation $R$ is the investigation of the multiplicity.

A closely related question is to determine whether there is any preference in the various direct sum decompositions of $V$ into invariant and irreducible subspaces. For the Heisenberg groups this question has been investigated in [1].

A special feature of the multiplicity problem in our situation is that the solution of the multiplicity problem is found by explicitly finding a direct sum decomposition for the unitary representation. The Hilbert space $V$ will be a function space closely related to the given solvable group $S$. The internal structure of the group $S$ is then employed directly to find such a decomposition. For greater details and generaltiy see [1].

The main topic of this paper is to associate to a binary integral quadratic form, at least of the kind mentioned previously, a solvable group and a unitary representation of this solvable group. The multiplicity problem of the form is then seen to be equivalent to the multiplicity problem of the corresponding unitary representation. Loosely speaking, the solvable group is constructed in such a way as to contain information about the automorphs or unit group of the form, and the representation constructed uses that information to reformulate the multiplicity problem of the form in terms of the multiplicity problem for the representation. This investigation is carried out in $\$ 2$.

The relationship between binary quadratic forms and quadratic fields indicates another method clearly equivalent to attaching fields which motivates the construction in $\S 3$. In this case a solvable group and a unitary representation are attached to a real quadratic field. Analogous to the previous situation, the solvable group contains information about the group of units of the real quadratic field and their action of this group as a group of linear automorphisms of the field. The ideals of the quadratic field, more specifically, the fractional ideals, pick out in a natural fashion a particular direct sum decomposition for the corresponding unitary representation.

Finally, in $\S 4$, we associate to a real quadratic field "the Laplacian" (see [2]) of the corresponding unitary representation. As indicated by Theorem 2.3 of [2], the eigenvalues of the Laplacian may play a role in the structure of the given real quadratic field.

The author wishes to thank John Brezin for his many helpful discussions and suggestions. Also to be thanked is the Institute of Advanced Study at Princeton for the opportunity of spending a year in relative peace.

2. Preliminaries. In this section we review some of the notions of quadratic form theory and unitary presentation theory needed for what follows. 
A reader familiar with both topics can easily skip to the next section as nothing but the most elementary facts are required.

Let $V$ be the space of all real binary quadratic forms. For $F\left(X_{1}, X_{2}\right)=$ $\alpha X_{1}^{2}+\beta X_{1} X_{2}+\gamma X_{2}^{2}$ in $V$, the symmetric matrix, also denoted by $F$, defined by

$$
F=\left(\begin{array}{cc}
\alpha & \beta / 2 \\
\beta / 2 & \gamma
\end{array}\right)
$$

is called the matrix of the quadratic form $F\left(X_{1}, X_{2}\right)$. The determinant $d=$ $\operatorname{det} F$ is called the determinant of the form. The quadratic form $F$ is called singular or nonsingular as $d=0$ or $d \neq 0$ respectively. Conversely, if $F=$ $\left(\begin{array}{cc}\alpha / 2 & \beta / 2 \\ \beta / 2\end{array}\right)$ is a real symmetric 2 by 2 matrix then the expression

$$
F\left(X_{1}, X_{2}\right)=X^{\prime} F X \text {, where } X=\left(\begin{array}{c}
X_{1} \\
X_{2}
\end{array}\right) \text { and } X^{\prime}=\left(X_{1} X_{2}\right)
$$

is its transpose, defines a binary quadratic form, and $F$ is the matrix of the quadratic form. In this way there is a vector space isomorphism from the space of all real binary quadratic forms onto the space of all real 2 by 2 symmetric matrices. We shall assume without explicit comment this identification in all that follows. The group $\operatorname{SL}(2, R)$ of real 2 by 2 matrices of determinant 1 with real coefficients acts on the space $V$ according to the following rule. For $T \in \mathrm{SL}(2, R)$ and $F$ in $V$ we put $F^{T}$ equal to the quadratic form with matrix $T^{\prime} F T$, where $T^{\prime}$ denotes the transpose of $T$.

For any $F$ in $V$ we define the orthogonal group of $F$ to be the collection of all $T$ in $\operatorname{SL}(2, R)$ satisfying $F^{T}=F$. It is a classical result that the orthogonal group of $F$ is an abelian algebraic group of dimension 1. In fact it is easy to show that there is a nonsingular matrix $S$ such that $S^{\prime} F S=E=$ $\left(\begin{array}{ll}1 & 0 \\ 0 & 1\end{array}\right)$ or $\left(\begin{array}{cc}1 & 0 \\ 0 & -1\end{array}\right)$. Then the orthogonal group of $E$ is conjugate to the orthogonal group of $F$. Thus we may just as well assume that $F=\left(\begin{array}{cc}1 & 0 \\ 0 & 1\end{array}\right)$ or $\left(\begin{array}{cc}1 & 0 \\ 0 & -1\end{array}\right)$. In the first case the orthogonal group of $F$ is given by $\left(\begin{array}{cc}a & b \\ -b & a\end{array}\right)$, where $a^{2}+$ $b^{2}=1$, and in the second case the orthogonal group of $F$ is given by $\left(\begin{array}{ll}a & b \\ b & a\end{array}\right)$, where $a^{2}-b^{2}=1$. Both are clearly abelian. The first is isomorphic to the circle and, hence, has dimension 1. The second is isomorphic to the hyperbola $x^{2}-y^{2}=1$ and, hence, consists of two components each having dimension 1 .

Suppose now that $F$ is an integral binary quadratic form, i.e., $F\left(X_{1}, X_{2}\right)$ $=a X_{1}^{2}+b X_{1} X_{2}+c X_{2}^{2}$, where $a, b, c$ are all integers. Let $G$ be the orthogonal group of $F$. By the unit group of $F$, also called the group of automorphs of $F$, we mean the group $G_{Z}$ of all integral matrices in.G. Clearly 
$G_{Z}$ is a discrete subgroup of $G$. We shall now assume in all that follows that $-4 d$ is not the square of some integer, where as before $d=\operatorname{det} F$. We state the following well-known theorem for future reference.

THEOREM A. Let $F$ be an integral binary quadratic form. We assume that $F$ is a nondegenerate form and $-4 d$ is not the square of some integer. Then $G / G_{Z}$ is compact.

Although we shall not prove this classical theorem we want to discuss a few facts about it. In case $d<0$ there is nothing to prove since $G$ is compact. The form $X^{2}-Y^{2}$ has a noncompact orthogonal group and finite unit group. Thus the condition that $-4 d$ not be a perfect square is certainly necessary. Under this latter assumption the fact that $G / G_{Z}$ is compact may be viewed as the classical theorem of Dirichlet on the group of units of a quadratic field. For further reference see [4].

We shall now discuss the tools needed from representation theory. What is essential for our purposes is simply a criterion for determining when a given unitary representation of a particular kind of solvable Lie group is irreducible. For a reference to the general theory see [3].

Let $G$ be a locally compact topological group given as the semidirect product $G=N \Varangle H$, where $N$ is an abelian group and $H$ is an arbitrary group. Let $\chi$ be a character on $N$. We say that the character $\chi$ has property (P) whenever the following condition is satisfied.

(P) For every $h$ in $H, \chi \neq \chi^{h}$, where $\chi^{h}$ is the character of $N$ defined by $\chi^{h}(x)=\chi\left(h^{-1} x h\right)$ for all $x$ in $N$.

The representations of $G$ which we are interested in come from inducing from characters on $N$. In fact in our case all the irreducible representations of $G$ can be described as representations induced from characters on $N$. Let us recall the definition of induced representations in our special case. If $\chi$ is a character of $N$, then the induced representation $U^{X}$ of $G$ coming from $\chi$ is defined by the rule

$$
U^{x}(n, h)(f)(\bar{h})=\chi(\bar{h}(n)) f(h+\bar{h}), \quad \bar{h} \in H,
$$

where $n$ is in $N, h$ is in $H$ and $f$ is in $L^{2}(H)$. Note that the space of the representation is $L^{2}(H)$. We now state the following special case of a theorem of Mackey.

TheOREM $\mathrm{M}_{1}$. Let $G$ be a locally compact topological group given as the semidirect product $G=N \Varangle H$, where $N$ is an abelian group and $H$ is any 
group. Let $\chi$ be a character on $N$ having property (P). Then the induced representation $U^{\chi}$ of $G$ coming from $\chi$ is an irreducible representation.

The second fact from representation theory is some procedure to determine when two representations of a group $G$ are unitarily equivalent. In particular, we shall want to know when two induced representations are unitarily equivalent. Again the source is Mackey [3].

THEOREM $\mathrm{M}_{2}$. Let $G$ be a locally compact topological group given as a semidirect product $G=N \Varangle H$, where $N$ is an abelian group and $H$ is an arbitrary group. Let $\chi_{1}, \chi_{2}$ be two characters on $N$ both satisfying property (P) and let $U^{\chi_{1}}$ and $U^{\chi_{2}}$ be the corresponding induced representations coming from $\chi_{1}$ and $\chi_{2}$ respectively. Then whenever $\chi_{1}=\chi_{2}^{h}$ for some $h$ in $H$, the corresponding induced representations $U^{\chi_{1}}$ and $U^{\chi_{2}}$ are unitarily equivalent.

Summarizing, we have that inducing to $G$ characters on $N$ having property (P) gives irreducible representations of $G$, and, further, if two such characters $\chi_{1}$ and $\chi_{2}$ of $N$ are on the same ' $H$-orbit', i.e., $\chi_{1}=\chi_{2}^{h}$ for some $h$ in $H$, then the corresponding induced representations of $G, U^{\chi_{1}}$ and $U^{\chi_{2}}$, are unitarily equivalent.

3. Quadratic forms and representation theory. In this section we associate to an integral binary quadratic form $F\left(X_{1}, X_{2}\right)$ a solvable Lie group $S$ and a discrete uniform subgroup $L$ of $S$. The main result relates the multiplicity problem of the form $F$ to the multiplicity problem of the right regular representation of $S$ on $L^{2}(L \backslash S)$.

Let $F\left(X_{1}, X_{2}\right)=a X_{1}^{2}+b X_{1} X_{2}+c X_{2}^{2}$ be an integral binary quadratic form. Denote the orthogonal group of $F$ by $G$ and the unit group of $F$ by $G_{Z}$. Then, under the assumptions on $F$ stated above, we have that $G / G_{Z}$ is compact. Define the solvable group $S=R^{2} \Varangle G$ and the subgroup $L=Z^{2}$ $\Varangle G_{Z}$. The semidirect product is defined relative to the natural action of $G$ on $R^{2}$ as a group of linear isomorphisms of $R^{2}$ relative to the standard basis $(1,0)$ and $(0,1)$ of $R^{2}$. Since $G / G_{Z}$ is compact, it is clear that $L \backslash S$ is compact. Hence, $L$ is a discrete uniform subgroup of $S$. There is a unique probability measure on the Borel sets in $L \backslash S$ which is invariant under the action of $S$ on $L \backslash S$ by right translations. Fixing $D_{1}=$ the unit square in $R^{2}$ as the fundamental domain for $R^{2} / Z^{2}$ and $D_{2}$ as the fundamental domain for $G / G_{Z}$, we fix this measure so that the measure of $D_{1}$ and $D_{2}$ is one. Define the Hilbert space $V=L^{2}(L \backslash S)$ of square integrable functions on $L \backslash S$ relative to this measure. It is a standard fact that 


$$
|f|_{V}^{2}=\int_{D_{1}} \int_{D_{2}}|f(x, t)|^{2} d t d x
$$

for all $f$ in $V$. Denote by $R$ the right regular representation of $S$ on $V$ given by $R_{s} f(L \bar{s})=f(L s \bar{s})$ for all $s$ and $\bar{s}$ in $S$. Clearly the invariance of the measure under right translations by $S$ implies that $R$ is a unitary representation of $S$ on $L^{2}(L \backslash S)$. We also require notation for the left regular representation of $S$ on the space of functions defined on $S$. Set $\left(L_{s} f\right)(s)=f\left(s^{-1} \bar{s}\right)$, where $S$ and $\bar{s}$ are in $S$ and $f$ is a function on $S$. The space $V$ can be identified with the collection of all functions $f$ on $S$ such that $L_{s} f=f$ for all $s$ in $L$, and $f$ considered as a function on $L \backslash S$ is in $V$.

Let $W$ be the space of all expressions

$$
f(x, t)=\sum_{n \in Z^{2}} f_{n}(t) \exp \{2 \pi i n \cdot x\},
$$

where the $f_{n}(t)$ are taken to be functions on $G$ which satisfy the following conditions.

1. $f_{n}(s+t)=f_{s^{\prime}(n)}(t)$ for all $s$ in $G_{z}$ and $t$ in $G$. The multiplication in $G$ has been written additively.

2. $\Sigma_{n \in Z^{2}} \int_{D_{2}}\left|f_{n}(t)\right|^{2} d t<\infty$.

The space $W$ is made into a Hilbert space by the following inner product. For

$f(x, t)=\sum_{n \in Z^{2}} f_{n}(t) \exp \{2 \pi i n \cdot x\}$ and $g(x, t)=\sum_{n \in Z^{2}} g_{n}(t) \exp \{2 \pi i n \cdot x\}$,

we set

$$
(f, g)=\sum_{n \in Z^{2}} \int_{D_{2}} f_{n}(t) \overline{g_{n}(t)} d t .
$$

It follows from [2] that the space $V$ can be identified with $W$. The identification is established as follows. Let $f$ be in $V$. For each $t$ in $G$ the map $x \rightarrow f(x, t)$ defines a square integrable function on $T^{2}=R^{2} / Z^{2}$. Define

$$
f_{n}(t)=\int_{T^{2}} f(x, t) \exp \{-2 \pi i n \cdot x\} d x .
$$

Then since $L_{s} f=f$ for all $s$ in $L$, it is easy to see that the functions $f_{n}(t)$ of $G$ satisfy condition 1 in the definition of $W$. From

$$
|f|_{v}^{2}=\int_{D_{1}} \int_{D_{2}}|f(x, t)|^{2} d x d t
$$

it follows that

$$
|f|_{v}=\left|f^{*}\right|_{L^{2}\left(T^{2} \times G / G_{Z}\right)}
$$


where $f^{*}$ is the function on the product of the groups $T^{2}$ and $G / G_{Z}$ defined from the restriction of $f$ to $D_{1}$ and to $D_{2}$. Since

$$
f_{n}(t)=\int_{T^{2}} f^{*}(x, t) \exp \{-2 \pi i n \cdot x\} d x
$$

for all $n$ in $Z^{2}$ it follows that

$$
|f|_{v}^{2}=\sum_{n \in Z^{2}} \int_{D_{2}}\left|f_{n}(t)\right|^{2} d t
$$

The process is clearly reversible.

Thus the map $f \rightarrow \Sigma_{n \in Z^{2}} f_{n}(t) \exp \{2 \pi i n \cdot x\}$ is a isometry of the Hilbert space $V$ onto the Hilbert space $W$. We shall without explicit comment make this identification in all that follows. In particular, the representation $R$ of $S$ on $V$ becomes the unitary representation of $S$ on $W$ given as follows:

$$
\begin{array}{r}
R_{(\bar{x}, \bar{t})}\left(\sum_{n \in Z^{2}} f_{n}(t) \exp \{2 \pi i n \cdot x\}\right) \\
=\sum_{n \in Z^{2}} f_{n}(t+\bar{t}) \exp \left\{2 \pi i t^{\prime}(n) \cdot \bar{x}\right\} \exp \{2 \pi i n \cdot x\}
\end{array}
$$

for all $(\bar{x}, \bar{t})$ in $S$.

For any integer $v \neq 0$, set $\Lambda(v)=\left\{n \in Z^{2}: F(n)=v\right\}$. The following well-known lemma implies that every point of $\Lambda(v)$ lies on the same $G$-orbit.

LEMMA 2. Take $x$ in $R^{2}$ and $v$ in $R^{\times}$such that $F(x)=v$. Then

$$
G \cdot x=\{t(x): t \text { in } G\}=\left\{y \in R^{2}: F(y)=v\right\} .
$$

PRoof. There is no loss in generality assuming that $F=\left(\begin{array}{ll}1 & 0 \\ 0 & 1\end{array}\right)$ or $F=\left(\begin{array}{rr}1 & 0 \\ 0 & -1\end{array}\right)$. In the first case the orthogonal group $G$ of $F$ is given by $G=\left(\begin{array}{cc}a & b \\ -b & a\end{array}\right)$, where $a^{2}+b^{2}=1$. Then if $y=\left(y_{1}, y_{2}\right)$ in $R^{2}$ satisfies $F(y)$ $=v$, we can form the matrix

$$
\left(\begin{array}{cc}
y_{1} & y_{2} \\
-y_{2} / v & y_{1} / v
\end{array}\right)
$$

which clearly is in $G$ and takes $(1,0)$ onto $y$. The second case is treated in exactly the same way.

Let $v \neq 0$ be an integer. Define $H(v)$ to be the space of all expressions $\Sigma_{n \in \Lambda(v)} f_{n}(t) \exp \{2 \pi i n \cdot x\}$ where the $f_{n}(t)$ are taken to be functions on $G$ which satisfy the following two conditions. 
1. $f_{n}(s+t)=f_{s^{\prime}(n)}(t)$ for all $n$ in $\Lambda(v), s$ in $G_{Z}$ and $t$ in $G$.

2. $\Sigma_{n \in \Lambda(v)} \int_{D_{2}} \mid f_{n}(t)^{2} d t<\infty$.

Lemma 3. The space $H(v)$ is an R-invariant subspace of $V$ and $V=$ $\Sigma_{v \in Z} \bigoplus H(v)$.

Proof. Clearly $H(v)$ is a subspace of $V$. Let

$$
f(x, t)=\sum_{n \in \Lambda(v)} f_{n}(t) \exp \{2 \pi i n \cdot x\}
$$

and $(\bar{x}, \bar{t})$ be in $S$. Then

$$
\left(R_{(\bar{x}, \bar{t})} f\right)(x, t)=\sum_{n \in \Lambda(v)} f_{n}(t+\bar{t}) \exp \left\{2 \pi i t^{\prime}(n) \cdot \bar{x}\right\} \exp \{2 \pi i n \cdot x\} .
$$

Setting $g_{n}(t)=f_{n}(t+\bar{t}) \exp \left\{2 \pi i t^{\prime}(n) \cdot \bar{x}\right\}$ we get that

$$
\left(R_{(\bar{x}, \bar{t})} f\right)(x, t)=\sum_{n \in \Lambda(v)} g_{n}(t) \exp \{2 \pi i n \cdot x\},
$$

where the functions $g_{n}$ satisfy conditions 1 and 2 above. The statement that $V=\Sigma \bigoplus H(v)$ follows immediately after noting that $Z^{2}$ is the disjoint union of the sets $\Lambda(v)$ and the definition of the space $V$.

The space $H(v)$ will be called the $v$ th multiplicity space. The remainder of the section is devoted to showing that $H(v)$ is a multiplicity space in the sense of [2], i.e. it is the direct sum of a finite number of unitarily equivalent subspaces, and to relate this number to the form $F$.

The group $G_{Z}$ acts on $Z^{2}$ and takes $\Lambda(v)$ onto itself. Hence we can consider the space of $G_{Z}$-orbits of $\Lambda(v)$, which we denote by $\Lambda(v) / G_{Z}$. Set $r(v)=$ the number of elements in $\Lambda(v) / G_{Z}$.

Let $n_{1}, \cdots, n_{r(v)}$ be a complete system of representatives in $\Lambda(v)$ of $\Lambda(v) / G_{Z}$. Thus $\Lambda(v)$ is the disjoint union of the sets $G_{Z} \cdot n_{1}, \cdots, G_{Z} \cdot$ $n_{r(v)}$, where $G_{Z} \cdot n=\left\{T(n): T \in G_{Z}\right\}$ denotes the $G_{Z}$-orbit at $n$.

The following definition takes into account the fact that for an expression $f=\Sigma_{n \in Z^{2}} f_{n}(t) \exp \{2 \pi i n \cdot x\}$ to be in $V$, the functions $f_{n}$ are related solely on $G_{Z}$-orbits.

For each $j=1, \cdots, r(v)$ define the subspace $H(v, j)$ to consist of the collection of all functions $f$ in $V$ given by

$$
f=\sum_{s \in G} f^{*}(t+s) \exp \left\{2 \pi i s^{\prime}\left(n_{j}\right) \cdot x\right\},
$$

where $f^{*}(t)$ is in $L^{2}(G)$.

Lemma 4. The space $H(v, j)$ is an R-invariant subspace of $H(v)$ independent of the choice of $n_{j}$ in $G_{Z} \cdot n_{j}$. Also we have $H(v)=\Sigma_{j=1}^{r(v)} \bigoplus H(v, j)$. 
Proof. Clearly $H(v, j)$ is a subspace of $H(v)$ and is independent of the choice of $n_{j}$ in $G_{Z} \cdot n_{j}$. To prove that $H(v, j)$ is $R$-invariant take $(\bar{x}, \bar{t})$ in $S$ and

$$
f(x, t)=\sum_{s \in G_{Z}} f^{*}(t+s) \exp \left\{2 \pi i s^{\prime}\left(n_{j}\right) \cdot x\right\}
$$

with $f^{*}(t)$ in $L^{2}(G)$. Assume that $f(x, t)$ is in $H(v, j)$. It follows that

$$
\left(R_{(\bar{x}, \bar{t})} f\right)(x, t)=\sum_{s \in G_{Z}} g^{*}(t+s) \exp \left\{2 \pi i s^{\prime}\left(n_{j}\right) \cdot x\right\},
$$

where $g^{*}(t)=f^{*}(t+\bar{t}) \exp \left\{2 \pi i t^{\prime}\left(n_{j}\right) \cdot x\right\}$. Clearly $g^{*}(t)$ is in $L(G)$. Thus the lemma is verified once we note that $\Lambda(v)$ is the disjoint union of the $G_{Z}$-orbits as mentioned before.

The next step is to show that each of the R-invariant subspaces $H(v, j)$ is an R-invariant and irreducible subspace of $H(v)$.

Lemma 5. For each $v \neq 0$ in $Z$ and $j=1, \cdots, r(v)$ the space $H(v, j)$ is an R-invariant and irreducible subspace of $H(v)$.

Proof. The lemma proceeds in two steps. Firstly using Theorem $M_{1}$ we construct an irreducible representation of $S$. Then we construct an intertwining operator between this irreducible representation of $S$ and $R \mid H(v, j)$, i.e., restriction of the operators of the representation $R$ to $H(v, j)$. For a more general discussion see [2].

Let $\chi$ be the character of $R^{2}$ defined by $\chi(x)=\exp \left\{2 \pi i n_{j} \cdot x\right\}$ for all $x$ in $R^{2}$. We claim that $\chi$ has property (P) with respect to $S$ and, hence, the representation $U^{X}$ of $S$ induced by $\chi$ is irreducible. To see that $\chi$ has property (P) suppose that $t$ is in $G$ and satisfies $\chi^{t}=\chi$. This implies that

$$
\chi(t(x))=\exp \left\{2 \pi i n_{j} \cdot t(x)\right\}=\exp \left\{2 \pi i t^{\prime}\left(n_{j}\right) \cdot x\right\}=\exp \left\{2 \pi i n_{j} \cdot x\right\}=\chi(x)
$$

for all $x$ in $R^{2}$. Then $t\left(n_{j}\right)=n_{j}$.

Choose $\tau$ in $\operatorname{GL}(2, R)$, the group of nonsingular 2 by 2 matrices over $R$, such that $\bar{t}=\tau t \tau^{-1}=\left(\begin{array}{ll}1 & x \\ 0 & 1\end{array}\right)$, where $x \neq 0$ in $R$. Then since $t F t^{\prime}=F$, we have $\bar{t} \bar{F} \bar{t}^{\prime}=\bar{F}$, where $\bar{F}=\tau F \tau^{\prime}$. We have to assume that the form $F$ is nonsingular, hence $\bar{F}$ is nonsingular. Write

$$
\bar{F}=\left(\begin{array}{ll}
\bar{\alpha} & \bar{\beta} \\
\bar{\beta} & \bar{\gamma}
\end{array}\right) .
$$

Then from $\bar{t} \bar{F} \bar{t}^{\prime}=\bar{F}$ we get $\bar{\gamma}=\bar{\beta}=0$, which contradicts the nonsingularity 
of $F$. Thus $\chi$ has property (P). Hence the representation $U^{x}$ of $S$ acting on the Hilbert space $L^{2}(G)$, given by

$$
\left(U_{(\bar{x}, \bar{t})}^{\mathrm{X}} f\right)(t)=\exp \left\{2 \pi i t^{\prime}\left(n_{j} \cdot \bar{x}\right)\right\} f(t+\bar{t})
$$

for all $f$ in $L^{2}(G)$ and $(\bar{x}, \bar{t})$ in $S$, is irreducible.

Next we note that the map $V(v, j): L^{2}(G) \rightarrow H(v, j)$ defined by

$$
(V(v, j) f)(x, t)=\sum_{s \in G_{Z}} f(t+s) \exp \left\{2 \pi i s^{\prime}\left(n_{j}\right) \cdot x\right\}
$$

for all $f$ in $L^{2}(G)$ and $(x, t)$ in $S$ is an intertwining operator for $R \mid H(v, j)$ and $U^{x n_{j}}$. Thus $H(v, j)$ is an R-invariant and irreducible subspace of $H(v)$.

THEOREM 1. Let $F$ be a nonsingular integral binary quadratic form. We assume that $-4 d$, where $d$ is the determinant of $F$, is not a perfect square. Let $G$ be the orthogonal group of $F$ and $G_{Z}$ the unit group of $F$. Set $S=$ $R^{2} \Varangle G, L=Z^{2} \Varangle G_{Z}$ and $R$ equal to the right regular representation of $S$ on $V$. For each integer $v \neq 0$ choose an $n$ in $Z^{2}$ such that $F(n)=v$. Then the space $H(v, n)$ of functions $f$ in $V$ of the form

$$
f(x, t)=\sum_{s \in G_{Z}} f^{*}(t+s) \exp \left\{2 \pi i s^{\prime}(n) \cdot x\right\}
$$

is an R-invariant and irreducible subspace of $V$ such that the multiplicity of $R \mid H(v, n)$ in $R$ is $r(v)$, the number of integral solutions of $F(x)=v$ in $Z^{2} / G_{Z}$

In particular, if $n_{1}, \cdots, n_{r(v)}$ define a complete system of representatives for $\Lambda(v) / G_{Z}$ then $V=\Sigma_{v} \bigoplus\left(\Sigma_{j=1}^{r(v)} H\left(v_{j} n_{j}\right)\right)$.

Proof. The theorem follows immediately from the preceding two lemmas and Theorem $\mathrm{M}_{2}$.

We also note that Theorem 1 could be modified to be a statement about the multiplicity of the irreducible representation induced from the character $\exp \{2 \pi i n \cdot x\}$ in $R$. We leave this equivalent formulation to the reader.

There are several simple variations possible in the preceding theorem which we now take note of. For example, assuming the situation in Theorem 1 since $G / G_{Z}$ is compact, it follows that $G^{0} / G_{Z} \cap G^{0}$ is also compact. The above theorem can then be modified by letting $G^{0}$ and $G^{0} \cap G_{Z}$ play the roles of $G$ and $G_{Z}$ respectively.

We close this section by giving two examples.

1. Let $F(X)=X_{1}^{2}+X_{2}^{2}$. Then the orthogonal group of $F$ is given by $G=\left(\begin{array}{cc}a & b \\ -b & a\end{array}\right)$, where $a^{2}+b^{2}=1$. The unit group is given by 


$$
G_{Z}=\left\{ \pm\left(\begin{array}{ll}
1 & 0 \\
0 & 1
\end{array}\right), \pm\left(\begin{array}{cc}
0 & -1 \\
1 & 0
\end{array}\right)\right\}
$$

Hence $S=R^{2}<T$ and $L=Z^{2}<G_{Z}$. For each of the integers $v$ and $n$ in $Z^{2}$ satisfying $F(n)=n_{1}^{2}+n_{2}^{2}=v$, the space $H(v, n)$ is given by the collection of all functions $f$ of the form

$$
f(x, t)=\sum_{s \in G_{Z}} f^{*}(t+s) \exp \left\{2 \pi i s^{\prime}(n) \cdot x\right\},
$$

where $f^{*}$ is in $L^{2}(T)$. The multiplicity of $R \mid H(v, n)$ in $R$ equals $1 / 4 \times$ the number of integral solutions of $F(X)=v$.

A possible variation is to let $S=R^{2} \Varangle T$ as before, but set $L=Z^{2}$. Then for $v$ in $Z$ and $n$ in $Z^{2}$ chosen so that $F(n)=v$, the space $H(v, n)$ is now the collection of all functions $f$ of the form $f(x, t)=f^{*}(t) \exp \{2 \pi i n \cdot x\}$, where $f^{*}$ is in $L^{2}(T)$ and the multiplicity of $R \mid H(v, n)$ in $R$ is the number of integral solutions of $F(X)=v$.

2. Let $F(X)=X_{1}^{2}-X_{1} X_{2}-X_{2}^{2}$. Since $A=\left(\begin{array}{ll}2 & 1 \\ 1 & 1\end{array}\right)$ is in $G_{Z}$, the unit group of $F$, and since $\operatorname{det} A=1$ and the eigenvalues of $A$ are real and positive, it follows that $G^{0}$, the connected component of the identity of $G$, the orthogonal group of $F$, is the one parameter subgroup through $A$. We recall that $G$ has dimension one and $G^{0}$ is a subgroup of index two in $G$. Moreover, it is easy to show that $A$ generates $G_{Z} \cap G^{0}$. Let $\hat{A}$ denote the group generated by $A$. Set $S=R^{2}<G^{0}$ and $L=Z^{2}<\hat{A}$. Take $v$ in $Z^{\times}, n$ in $Z^{2}$ satisfying $F(n)=v$. In this case we have the decomposition $\Lambda(v)=G \cdot n$ $=G^{0} \cdot n \cup G^{0} \cdot m$, where $m$ is in $Z^{2}, m$ is not in $G^{0} \cdot n$ and $F(m)=v$. Thus the multiplicity of the irreducible representation $U^{\chi_{n}}$ of $S$ induced by the character $\chi_{n}(x)=\exp \{2 \pi i n \cdot x\}$ of $R^{2}$ is equal to $r(n)=\# G^{0} \cdot n / G_{Z}$ $\cap G^{0}$. Thus $r(n)$ is equal to $1 / 2$ times the number of nonassociated solutions of the equation $F(X)=F(n)$. The term nonassociated is used as usual to mean the number of equivalence classes in $G^{0}$ modulo the action of the group $G_{Z}$.

4. Quadratic fields and solvable groups. In this section we reformulate the construction of the previous section replacing the notion of a quadratic form by the equivalent notion of a quadratic field. This will be done solely for real quadratic fields.

Let $K$ be a real quadratic field. Denote by 0 the ring of algebraic integers of $K$ and by $U^{+}$the group of totally positive units in 0 . We note that in all that follows, the ring 0 could be replaced by any ideal of 0 and the group $U^{+}$by any subgroup of $U^{+}$. 
Write $K=Q(\sqrt{ } d)$, where $d$ is the discriminant of the quadratic field $K$. Let 1 and $w$ be the integral basis of $K$ defined by

$$
w= \begin{cases}\sqrt{d} / 2, & d \equiv 0 \bmod 4, \\ 1+\sqrt{d} / 2, & d \equiv 1 \bmod 4\end{cases}
$$

In terms of this integral basis, the norm function $N$ of $K$ and the trace function $\operatorname{Tr}$ of $K$ can be written as

$$
\begin{aligned}
& N(r+s w)= \begin{cases}r^{2}-(d / 4) s^{2}, & d \equiv 0 \bmod 4, \\
r^{2}+r s+((1-d) / 4) s^{2}, & d \equiv 1 \bmod 4,\end{cases} \\
& \operatorname{Tr}(r+s w)= \begin{cases}2 r, & d \equiv 0 \bmod 4, \\
2 r+s, & d \equiv 1 \bmod 4 .\end{cases}
\end{aligned}
$$

By the Dirichlet unit theorem the group $U^{+}$is infinite cyclic and, hence, isomorphic to $Z$. Let $\epsilon$ denote the generator of $U^{+}$. The group $U^{+}$acts as a group of $Q$-vector space automorphisms of $K$. For $t$ in $U^{+}$, the corresponding $Q$-vector space automorphism is given by $x \rightarrow t \cdot x$. Since the eigenvalues of the element considered as a $Q$-vector space isomorphism of $K$ are both positive, it follows that $U^{+}$lies on a unique one parameter group $U_{R}^{+}$of $R$-vector space automorphisms of $K_{R}=K \otimes_{Q} R$. The ring 0 defines a lattice in $K_{R}$ which is preserved by $U^{+}$. Hence $U^{+}$is a discrete uniform subgroup of $U_{R}^{+}$, and relative to any basis of $K_{R}$ coming from an integral basis of 0 we have that $U^{+}$consists of integer matrices.

Let $S_{3}$ denote the unique three-dimensional simply connected solvable analytic group having the property that $\operatorname{ad}_{N} S_{3}$ is not compact. By $N$ we mean here the nilradical of $S_{3}$, i.e., the maximal connected normal nilpotent subgroup of $S_{3}$. In the case of $S_{3}$ we have that $N=R^{2}$ and that $S_{3}=N<$ $R$, where $R$ acts faithfully on $N$ as a group of automorphisms.

Set $L=0 \Varangle U^{+}$and $S_{3}=K_{R} \Varangle U_{R}^{+}$. The group $L$ is a discrete uniform subgroup of $S_{3}$ by our comments above. Also note that this construction is analogous to the situation in the previous section where we dealt with the connected component of the orthogonal group of an integral binary quadratic form with negative determinant.

The group $S_{3}$ can be described in terms of any real quadratic field. The choice of one real quadratic field to describe $S_{3}$ determines a choice of a discrete uniform subgroup $L$ of $S_{3}$. In fact, we get a family of such discrete uniform subgroups $L$ of $S_{3}$ if we allow for the replacement of $O$ by any ideal of 0 and of $U^{+}$by any nontrivial subgroup of $U^{+}$. 
A final word before we begin the analogous investigation to that considered in §3. Since for $x$ in $K$ the map $y \rightarrow x \cdot y$ of $K$ is a $Q$-linear map of $K$, it extends to an $R$-linear map of $K_{R}$. Thus the notation $x \cdot y$ is well defined for $x$ in $K$ and $y$ in $K_{R}$. Also the trace function on $K$ and the norm function on $K$ extend naturally to $K_{R}$. Denote the extensions by the same symbols used before.

The conventions of measure introduced in $\$ 3$ are maintained here without further comment. Let $R$ denote the right regular representation of $S_{3}$ on $V=L^{2}(L \backslash S)$. The left regular representation of $S_{3}$ on the space of functions defined on $S_{3}$ is still denoted by $L$.

The program in the following few lemmas is to translate into the language of quadratic fields the work of the previous section. This being the case, we shall not give complete details in our arguments but only indicate the translation.

The first step in the translation is to characterize in terms of the trace map the characters of $K_{R}$ which vanish on 0 .

LEMma 5. The characters of $K_{R}$ which vanish on 0 are of the form $x \rightarrow \exp \{2 \pi i \operatorname{Tr}(y \cdot x)\}$ for fixed $y$ in $D$, where $D$ consists of all those $y$ in $K$ such that $\operatorname{Tr}(y \cdot 0) \subset Z$.

Proof. Clearly for $y$ in $D$ the map $x \rightarrow \exp \{2 \pi i \operatorname{Tr}(y \cdot x)\}$ is a character of $K_{R}$ which vanishes on 0 .

Let $\chi$ be a character on $K_{R}$ which vanishes on 0 . Then writing $\chi$ relative to the integral basis 1 and $w$, introduced above for $K$, we have $\chi(r+s w)=\exp \{2 \pi i(n \cdot r+m \cdot s)\}$, where $n$ and $m$ are in $Z$. It is a simple computation to show that $\chi(x)=\exp \{2 \pi i \operatorname{Tr}(y \cdot x)\}$, where $y$ is given by $y=(n / 2,2 m / d)$ whenever $d \equiv 0 \bmod 4$, and $y=(n-m / d, 2 m-n / d)$ whenever $d \equiv 1 \bmod 4$. This completes the proof of the lemma.

The set $D$ is a lattice in $K$. The following lemma tells us a little more.

LEMMA 6. Every element $y$ in $D$ has the property that $N(y)$ is in $(1 / d) Z$.

Proof. The condition that $\operatorname{Tr}(y \cdot 0) \subset Z$ is equivalent to the requirement that when $y=y_{1}+y_{2} w$ we have $2 y_{1}$ and $\left(y_{2} d / 2\right)$ in $Z$ for $d \equiv 0$ $\bmod 4$, and $2 y_{1}+y_{2}$ and $y_{1}+((d+1) / 2) y_{2}$ in $Z$ when $d \equiv 1 \bmod 4$. In the first case, $N(y)=y_{1}^{2}-\left(y_{2}^{2} / 4\right) d$ and $\bar{y}=y_{2} d / 2+2 y_{1}(\sqrt{ } d / 2)$ is an algebraic integer. Hence the norm of $y$, which equals $d \cdot N(y)$, is an integer. The second case is handled in the same way. Thus the lemma is verified.

Let $W$ be the space of all expressions

$$
f(x, t)=\sum_{y \in D} f_{y}(t) \exp \{2 \pi i \operatorname{Tr}(y \cdot x)\},
$$


where the $f_{y}(t)$ are meant to be functions on $U_{R}^{+}$satisfying the following conditions.

1. $f_{s \cdot y}(t)=f_{y}(s+t)$ for all $s$ in $U^{+}, t$ in $U_{R}^{+}$, and where the multiplication in $U_{R}$ has been written additively.

2. $\Sigma_{y \in D} \int_{D_{2}}\left|f_{y}(t)\right|^{2} d t<\infty$, where $D_{2}$ is a fundamental domain of $U_{R}^{+}$with respect to $U^{+}$as before.

The space $W$ is made into a Hilbert space by defining the following inner product:

$$
(f, g)=\sum_{y \in D} \int_{D_{2}} f_{y}(t) \overline{g_{y}(t)} d t,
$$

where $f=\Sigma_{y \in D} f_{y}(t) \exp \{2 \pi i \operatorname{Tr}(y \cdot x)\}$, and $g=\Sigma_{y \in D} g_{y}(t) \exp \{2 \pi i \operatorname{Tr}(y \cdot x)\}$.

We state the following lemma whose proof is the same as the corresponding lemma in the previous section.

Lemma 7. The map $f(x, t) \rightarrow \Sigma_{y \in D} f_{y}(t) \exp \{2 \pi i \operatorname{Tr}(y \cdot x)\}$, defined by setting

$$
f_{y}(t)=\int_{D_{1}} f(x, t) \exp \{-2 \pi i \operatorname{Tr}(y \cdot x)\} d x,
$$

where $D_{1}$ is a fundamental domain for $R^{2}$ relative to $Z^{2}$ as before, is a unitary operator of $V$ onto $W$.

The above identification of $V$ and $W$ shall be implicitly assumed in all that follows.

For each $v$ in $Z^{+}$set $\Lambda(w)=\{x$ in $d: N(x)=v / d\}$. Observe that $\Lambda(v)$ is not contained in one $U_{R}$-orbit. Set

$$
\Lambda^{+}(v)=\{x \text { in } \Lambda(v): x>0\} \text { and } \Lambda^{-}(v)=\{x \text { in } \Lambda(v): x<0\} .
$$

Then $\Lambda(v)=\Lambda^{+}(v) \cup \Lambda^{-}(v)$ and $\Lambda^{+}(v)$ lies on a single $U_{R^{+}}^{+}$orbit. The verification follows exactly as before.

The next lemma translates Lemma 3. The proof is left to the reader.

LEMMA 8. The space $H^{+}(v)$ consisting of all expressions of the form

$$
f(x, t)=\sum_{y \in \Lambda^{+}(v)} f_{y}(t) \exp \{2 \pi i \operatorname{Tr}(y \cdot x)\},
$$

where the $f_{y}(t)$ are taken as functions on $U_{R}^{+}$satisfying the following two conditions.

1. $f_{s . y}(t)=f_{y}(s+t)$ for all $t$ in $U_{R}^{+}, s$ in $U^{+}$and $y$ in $\Lambda^{+}(v)$.

2. $\Sigma_{y \in \Lambda^{+}(v)} \int_{D_{2}}\left|f_{y}(t)\right|^{2} d t<\infty$ is an R-invariant subspace of $V$ and, moreover, we have $V^{=}=\Sigma_{v} \oplus H^{+}(v) \oplus H^{-}(v)$. 
The ideals of $K$ will now be used to give a natural decomposition of the space $H^{+}(v)$ into the direct sum of $R$-invariant and irreducible subspaces in case $v \neq 0$.

Take $v$ in $Z^{\times}$. Then $U^{+}$acts on $\Lambda^{+}(v)$ and we denote the space of orbits by $\Lambda^{+}(v) / U^{+}$. Let $r^{+}(v)$ denote the number of elements in $\Lambda^{+}(v) / U^{+}$. Each $U^{+}$-orbit $V$ of $\Lambda^{+}(v)$ determines a unique fractional ideal $J(V)$ of $K$ defined by $J(V)=(1 / p) 0$, where $p$ is any element in $V$.

THEOREM 2. Let $V$ be in $\Lambda^{+}(v) / U^{+}$and $H^{+}(v, V)$ be the subspace of $V$ consisting of all those functions $f$ in $H^{+}(v)$ such that $L_{y} f=f$ for all $y$ in $J(V)$. Then $H^{+}(v, V)$ is an R-invariant and irreducible subspace of $\mathrm{H}^{+}(v)$. Moreover we have the following:

1. $R \| H^{+}(v, V)$ is unitarily equivalent to $R \| H^{+}(\bar{v}, \bar{V})$ is and only if $v=\bar{v}$.

2. $H^{+}(v)=\Sigma_{V} H^{+}(v, V)$, where the sum is taken over all $U^{+}$-orbits $V$ in $\Lambda^{+}(v)$.

3. The multiplicity of $R \mid H^{+}(v, V)$ in $R$ equals $r^{+}(v)$.

Proof. Let $V$ be a $U^{+}$-orbit in $\Lambda^{+}(v), J=J(V)$ the corresponding fractional ideal given by $J=(1 / p) O$ with $p$ in $V$. The theorem is a direct consequence of the claim that the space $H^{+}(v, V)$ consists of all those functions of the form

$$
f(x, t)=\sum_{s \in U^{+}} f^{*}(s, t) \exp \{2 \pi i \operatorname{Tr}(s \cdot p \cdot x)\},
$$

where $f^{*}(t)$ is in $L^{2}\left(U_{R}^{+}\right)$. This fact is verified as follows. For

$$
f(x, t)=\sum_{y \in \Lambda^{+}(v)} f_{y}(t) \exp \{2 \pi i \operatorname{Tr}(y \cdot x)\}
$$

in $H^{+}(v, V)$ we have $\left(L_{(1 / p) Z} f\right)(x, t)=f(x, t)$ for all $Z$ in 0 . It follows that $\operatorname{Tr}(y / p)$ is in $Z$. Since, moreover, $N(y)=N(p)$, it is clear that $y / p$ is in $U^{+}$. The statement characterizing $H^{+}(v, V)$ is verified and the theorem follows.

The direction of the preceding two sections has been to define a solvable Lie group and a discrete uniform subgroup from either a given quadratic form or quadratic field. We shall now show that every discrete uniform subgroup of $S_{3}$ can be defined essentially in this way.

We begin with an example. Let $A=\left(\begin{array}{ll}2 & 1 \\ 1 & 1\end{array}\right)$. The eigenvalues of the matrix $A$ are $3^{ \pm} \sqrt{5} / 2$ both real and positive. Hence $A$ lies on a one parameter group $A^{t}$ of matrices. Form the group $S_{3}$ described as the manifold $R^{2} \times R$ and whose group operation is given by

$$
(x, t)(\bar{x}, \bar{t})=\left(x+A^{t}(\bar{x}), t+\bar{t}\right) .
$$


Let $L$ be the discrete uniform subgroup formed by the set $Z^{2} \times Z$ of $S_{3}$. The first step is to give the group $S_{3}$ and its discrete uniform subgroup $L$ in terms of quadratic fields as before.

The eigenvalues of $A$ we denote by $\lambda, \lambda^{-1}$, where $\lambda$ is the greatest one. Let $K=Q(\sqrt{ } 5)$. Then $\lambda, \lambda^{-1}$ are totally positive units in $K$. Let $u_{1}=$ 1 and $u_{2}=\lambda-2$. Then $u_{1}$ and $u_{2}$ define an integral basis of 0 , and the matrix of the map $x \rightarrow \lambda x$ relative to the basis $u_{1}$ and $u_{2}$ is easily seen to be $A$. Finally, it is easy to see that the element $\lambda$ generates $U^{+}$, the group of totally positive units of $K$. Hence $S_{3}=K_{R} \Varangle U_{R}^{+}$and $L=0 \Varangle U^{+}$.

THEOREM 3. Let $S_{3}$ be the three-dimensional simply connected solvable analytic group such that $\operatorname{ad}_{N} S_{3}$ is not compact, where $N$ is the nilradical of $S_{3}$ as before. Let $L$ be a discrete uniform subgroup of $S_{3}$. Then there is a quadratic field $K$, an ideal $A$ of $O$, the ring of algebraic integers in $K$, and a nontrivial subgroup $U_{1}$ of $U^{+}$, the totally positive units of $K$, such that $L=A<U_{1}$ and $S_{3}=K_{R}<U_{R}^{+}$.

Proof. It is not hard to see that we can write $S_{3}=R^{2} \times R$ and $L=$ $Z^{2} \times Z$ as before. For details see [2]. Since $S_{3}$ is connected, $\operatorname{ad}_{S} L \subset \operatorname{SL}_{2}(Z)$. Hence $\operatorname{ad}_{{ }^{2}} L=\hat{A}$, where $A=\left(\begin{array}{ll}a & b \\ c & d\end{array}\right)$, where $a, b, c$ and $d$ are in $Z$ and $a d-b c=1$. The eigenvalues of $A$ can be written $\lambda, \lambda^{-1}=1 / 2\left(t^{ \pm} u \sqrt{ } d\right)$, where both $t$ and $u$ are in $Z$. Let $K=Q(\sqrt{ } d)$. Then $\lambda, \lambda^{-1}$ are totally positive units in $K$. The elements $u_{1}=b$ and $u_{2}=\lambda-a$ defined an integral basis of an ideal $A$ in $O$, and the matrix of the $Q$-linear map $x \rightarrow x$ of $K$ is $\left(\begin{array}{ll}a & b \\ c & d\end{array}\right)$ relative to this basis. Let $U_{1}$ be the subgroup of $U^{+}$generated by $A$. The theorem follows.

5. The Laplacian. Let $K=Q(\sqrt{ } d)$ be a quadratic field of discriminant $d>0,0$ its ring of algebraic integers and $U^{+}$the totally positive units. Form $S_{3}=K_{R} \Varangle U_{R}^{+}$and $L=0 \Varangle U^{+}$. Denote the right regular representation of $S_{3}$ on $V=L^{2}(L \backslash S)$ by $R$. The object of this section is to define 'a Laplacian' for the quadratic field using the representation $R$. For the more general situation see [2].

Take $v$ in $Z^{\times}$and let $V$ be a $U^{+}$-orbit in $\Lambda^{+}(v)$. Consider $H^{+}(v, V)$ as before. The subspace $H_{\infty}^{+}(v, V)$ consists of the collection of all $f$ in $H^{+}(v, V)$ where the corresponding $f^{*}(t)$ in the identification of the previous section are taken to be in $C^{\infty}\left(U_{R}^{+}\right) \cap L^{2}\left(U_{R}^{+}\right)$. Then $H_{\infty}^{+}(v, V)$ is a dense subspace of $\mathrm{H}^{+}(v, V)$ and every symmetric and nonnegative semidefinite differential operator of $H_{\infty}^{+}(v, V)$ extends to a selfadjoint operator of $H^{+}(v, V)$.

The first step in the definition of a Laplacian corresponding to the representation $R$ is to define operators on $H_{\infty}^{+}(v, V)$ corresponding to a basis of 
the Lie algebra of $S$. Recall 1 and $w$ are taken as an integral basis of $K$ as before.

Let $\underline{X}_{1}, \underline{X}_{2}$, and $\underline{X}_{3}$ be the operators of $H_{\infty}^{+}(v, V)$ given by

$$
\begin{aligned}
& \underline{X}_{1} f(x, t)=2 \pi i \sum_{s \in U^{+}} f^{*}(t+s) \operatorname{Tr}(t \cdot s \cdot p) \exp \{2 \pi i \operatorname{Tr}(s \cdot p \cdot x)\}, \\
& \underline{X}_{2} f(x, t)=2 \pi i \sum_{s \in U^{+}} f^{*}(t+s) \operatorname{Tr}(t \cdot s \cdot p \cdot w) \exp \{2 \pi i \operatorname{Tr}(s \cdot p \cdot x)\}, \\
& \underline{X}_{3} f(x, t)=\sum_{s \in U^{+}} \frac{d}{d t} f^{*}(t+s) \exp \{2 \pi i \operatorname{Tr}(s \cdot p \cdot x)\},
\end{aligned}
$$

where $f(x, t)=\Sigma_{s \in U^{+}} f^{*}(t+s) \exp \{2 \pi i \operatorname{Tr}(s \cdot p \cdot x)\}$ with $f^{*}(t)$ in $C^{\infty}\left(U_{R}^{+}\right)$ $\cap L^{2}\left(U_{R}^{+}\right)$.

The proof that the operator defined by $\Delta(v, V)=-\left(\underline{X}_{1}^{2}+\underline{X}_{2}^{2}+\underline{X}_{3}^{2}\right)$, a symmetric and nonnegative semidefinite differential operator of $H_{\infty}^{+}(v, V)$, is the same as in [2].

Finally, the explicit form of $\Delta$ is given by

$$
\Delta(v, V)=d^{2} / d t^{2}-g(t) \quad \text { where } g(t)=[\operatorname{Tr}(t \cdot p)]^{2}+[\operatorname{Tr}(t \cdot p \cdot w)]^{2} .
$$

\section{REFERENCES}

1. L. Auslander and J. Brezin, Fibre bundle structures and harmonic analysis on Heisenberg manifolds, Proc. Maryland Conference on Harmonic Analysis, Springer-Verlag, New York, 1973.

2. J. Brezin, Function theory on metabelian solvmanifolds, J. Functional Analysis 10 (1972), 33-51.

3. G. Mackey, Induced representations of locally compact groups. I, Ann. of Math. (2) 55 (1952), 101-139. MR 13, 434.

4. C. L. Siegel, Lectures on quadratic forms, Tata Institute of Fundamental Research Lectures on Math., no. 7, Tata Institute of Fundamental Research, Bombay, 1967. MR 42 \#5911.

DEPARTMENT OF MATHEMATICS, HERBERT H. LEHMAN COLLEGE (CUNY), NEW YORK, NEW YORK 10003

Current address: Department of Mathematics, University of Connecticut, Storrs, Connecticut 06250 